\title{
Crossover from Rate-Equation to Diffusion-Controlled $\underline{\text { Kinetics in Two-Particle Coagulation }}$
}

\author{
V. Privman, ${ }^{a}$ C.R. Doering ${ }^{a}$ and H.L. Frisch ${ }^{b}$ \\ ${ }^{a}$ Department of Physics, Clarkson University, Potsdam, New York 13699-5820 \\ ${ }^{b}$ Department of Chemistry, State University of New York, Albany, New York 12222
}

\begin{abstract}
We develop an analytical diffusion-equation-type approximation scheme for the one-dimensional coagulation reaction $A+A \rightarrow A$ with partial reaction probability on particle encounters which are otherwise hard-core. The new approximation describes the crossover from the mean-field rate-equation behavior at short times to the universal, fluctuation-dominated behavior at large times. The approximation becomes quantitatively accurate when the system is initially close to the continuum behavior, i.e., for small initial density and fast reaction. For large initial density and slow reaction, lattice effects are nonnegligible for an extended initial time interval. In such cases our approximation provides the correct description of the initial mean-field as well as the asymptotic large-time, fluctuation-dominated behavior. However, the intermediate-time crossover between the two regimes is described only semiquantitatively.
\end{abstract}

PACS numbers: $82.20 . \mathrm{Mj}$ and $05.40 .+\mathrm{j}$ 


\section{Introduction}

Reaction-diffusion systems in low dimensions have been investigated recently with emphasis on fluctuation-dominated effects, specifically, the breakdown of the standard chemical rate equations which correspond to the "mean-field" approximation of the reaction kinetics. Much recent interest has been focused on the simplest reactions of two-particle coagulation, $A+A \rightarrow A$, and annihilation, $A+A \rightarrow$ inert, on the onedimensional lattice [1-13]. Indeed, in the diffusion-limited, instantaneous-reaction case, these processes show non-mean-field power-law decay of the $A$-particle density in one dimension. Exact results have been derived indicating that the particle concentration per unit length, $c(t)$, decays according to

$$
c(t \text {-large }) \sim 1 / \sqrt{D t}
$$

where $D$ is the single-particle diffusion constant in the dilute limit, and $t$ is the time. Experimental verification of the theoretical predictions has been initiated recently [14].

The result (1.1) differs markedly from the prediction of the rate equation

$$
\frac{d c}{d t} \propto-c^{2}
$$

appropriate for these reactions. Indeed, the naive mean-field power law is simply

$$
c_{\text {mean-field }}(t \text {-large }) \sim \ell / D t
$$

where $\ell$ is the lattice spacing. Modified mean-field treatments can capture the diffusioncontrolled scaling in some cases [15]. 
Now if the reactions on particle encounters are not instantaneous but rather occur with probability $q$, where $0<q \leq 1$, then for slow reaction, $q \ll 1$, one might expect the mean-field expressions to apply initially although for large times a crossover to the fluctuation-dominated behavior (1.1) is expected. Extensive numerical studies of the annihilation reaction, $A+A \rightarrow$ inert, with partial reaction probability [16-17], indicate that there is a crossover from the mean-field to the fluctuation-dominated behavior. Similar crossover effects were observed in related dimer-deposition models with diffusional relaxation [18]. However, due to lack of any theoretical description of these crossover effects, the data interpretation [16-17] has been a fit to an ad-hoc analytical expression: a $t$-dependent-effective-exponent power law, with no conclusive results. In fact the analytical form used [16-17] is known to be wrong in other better understood mean-field to fluctuation crossovers in static critical phenomena [19], and it is also inconsistent with a certain dimensional-analysis scaling form [3] appropriate in the limit of vanishing initial density.

Approximate theoretical description of the mean-field to fluctuation-dominated crossover is important for several reasons. Firstly, it will provide guidance on the limits of validity of the rate-equation approximation and illuminate its microscopic foundations. Secondly, it will shed light on the universality of the large-time fluctuation dominated power-law behavior (1.1). Finally, it can suggest scaling forms and variablecombinations to use in actual data fits, as well as directions of improving the simplest mean-field approximation schemes in those regimes where mean-field rate equations do apply; see [20] for a discussion of multiparticle reactions for which data fits can be improved by a careful choice of the mean-field model variant.

This work reports a new diffusion-equation type approximation scheme for the description of the mean-field to fluctuation-dominated crossover in one-dimensional reactions. We consider the coagulation reaction, $A+A \rightarrow A$, rather than annihilation. 
Indeed, while these reactions have many common features [1-13], the former, coagulation reaction, is described for $q=1$ by a local diffusion equation for the interparticle distance, in the continuum limit [8]. This provides a convenient starting point.

When the particles do not react with probability 1 , their interactions upon encounters must be specified. We assume hard-core interactions in cases of no reaction; our model is defined in Section 2. It has been anticipated in the literature [21-22] that a diffusive description of the partial reaction probability system will involve the so-called "radiation" boundary conditions [23]. However, no specific implementations have been reported yielding results for one-dimensional reactions.

In fact, the form of the radiation boundary conditions turns out to be the least straightforward aspect of the problem; the radiation boundary conditions are discussed in detail in Section 3. Analytical results within our approximation scheme are derived in Section 4. Various limiting forms as well as comparison with numerical Monte Carlo data and concluding discussion are given in Section 5. 


\section{$\underline{\text { 2. Diffusive Approximation for Interparticle Distribution }}$}

We consider particles hopping on a line, each independently, and on pairwise encounters subject to reaction $A+A \rightarrow A$ with probability $q$, where $0 \leq q \leq 1$, and to hard-core interaction (i.e., the hopping attempt fails) with probability $1-q$. Let $c(t)$ denote particle concentration per unit length at time $t$, and

$$
c(0)=\rho
$$

be the initial concentration. The interparticle distribution will be denoted by $P(x, t)$. It is proportional to the probability density to find interparticle distance $x \geq 0$, at time $t \geq 0$. It will be normalized as follows:

$$
\int_{0}^{\infty} P(x, t) d x=\frac{c(t)}{\rho} .
$$

We consider a random initial distribution of particles

$$
P(x, 0)=\rho e^{-\rho x} .
$$

Our aim is to develop an approximate diffusion-type description of the time-evolution of the distribution $P$. Thus we assume that the time-dependence can be modeled by a probability balance equation of the form

$$
\frac{\partial P}{\partial t}=-\frac{\partial J}{\partial x} .
$$

The current $J$ will have the diffusive component due to particle hopping, 


$$
J_{\text {diff }}=-2 D \frac{\partial P}{\partial x} .
$$

Here $D$ is the single-particle diffusion constant in the dilute limit, i.e., when the hardcore constraint is ineffective in slowing down the particle hopping. It is well known that the interparticle distance then evolves with twice the particle diffusion constant.

In addition, the evolution of $P$ is affected by the reaction, which essentially enters via the boundary conditions at $x=0$, and by the hard-core constraint on the particle motion during those encounters which do not lead to reaction. Let us consider first the effect of the hard core interactions.

On each such hard-core encounter, illustrated by $x_{m}=0$ in Figure 1 , the two interparticle distances nearest to the distance between the two colliding particles, each cannot evolve purely diffusively but rather they are driven to shorten. In Figure 1, the interparticle distances $x_{m-1}$ and $x_{m+1}$ are thus correlated with each other and with the middle distance $x_{m}$, with the correlation being strongest when $x_{m}$ approaches zero. While the exact modeling of such correlations might be quite complicated and involve many-body effects, it can be modeled approximately, phenomenologically, by assuming a random (in time and in coordinate $x$ ) "driving force" pushing the $x$-distances toward the origin of the $x$-axis.

Thus we approximately model the hard-core effects by including a drift term in the current. The drift velocity is in the negative- $x$ direction, of magnitude conveniently denoted by $v=2 D(1-q) p(t)$ :

$$
J_{\text {hard-core }}=-2 D(1-q) p(t) P(x, t) \text {. }
$$

The proportionality to $1-q$ is suggested by the fact that this factor is the probability 
of the hard-core interaction rather than reaction, on encounter. The quantity $p(t)$ was introduced to represent the fact that the drift effect due to the hard-core interactions should be proportional to the density of particle pairs at contact, given by $P(0, t)$, which suggests that we put

$$
p(t)=P(0, t) .
$$

Finally, the dimensionless constant in (2.6) was determined by the observation that for no reaction (only hard-core interactions, $q=0$ ) the stationary solution for all times must be given by (2.3), while the sum of the diffusive and hard-core currents, generally given by

$$
J=-2 D\left[(1-q) p P+\frac{\partial P}{\partial x}\right],
$$

must be identically zero for $q=0$.

Let us introduce the reduced time variable

$$
\tau \equiv 2 D t
$$

Then we get the equations

$$
\begin{gathered}
\frac{\partial P}{\partial \tau}=(1-q) p \frac{\partial P}{\partial x}+\frac{\partial^{2} P}{\partial x^{2}} \\
p(\tau)=P(0, \tau)
\end{gathered}
$$

The first equation follows from (2.4) with (2.8). The solution is well defined only after the boundary conditions at $x=0$ are added. The boundary conditions are discussed 
in Section 3. Note however that our approximate equation is nonlinear. Thus we are going to solve it within an adiabatic approximation to be specified in Section 4. 


\section{Radiation Boundary Conditions and Universality}

At $x=0$, we assume time-dependent radiation boundary conditions [23],

$$
J(0, t)=-2 D a(t) P(0, t), \quad t>0,
$$

which correspond to partial absorption of the diffusers. Of course, in our case the "absorption" at the origin means the disappearance of an interparticle distance due to coagulation reaction. We will now address the issue of modeling the quantity $a(t)$ in (3.1).

Generally, the applicability of diffusion-type approximation schemes improves when the system is close to the continuum limit. Specifically, if the initial density is small as compared to the inverse of the microscopic lattice spacing, i.e., $\rho \ell \ll 1$, and the reaction is fast, $(1-q) \ll 1$, then the continuum approximation should become more accurate. Lattice effects are more significant for large particle densities, as well as for slow reactions since the latter are expected to be described by the mean-field (lattice-

dependent) expressions for extended times. For instantaneous reactions, $q=1$, the hard-core constraint is irrelevant and the description is in terms of an ordinary, linear diffusion equation, which then provides an extremely accurate approximation, exact in the continuum limit [8]. For a discrete-space modeling of the diffusion-controlled limit, see [24].

Thus we expect that our approximation scheme will also be more accurate for smaller densities and faster reactions. However, we cannot fully eliminate the latticespacing dependence because our aim is to describe the crossover from the latticedependent mean-field behavior for short times to the universal, continuum-limit, fluctuation dominated behavior at large times. 
In a lattice hopping-diffuser model, one can show that the quantity $a$ entering the radiation boundary conditions, is given by

$$
a_{\text {lattice }}=\frac{q}{(1-q) \ell}\left[1+O\left(\frac{v \ell}{D}\right)\right] \text {. }
$$

Here the absorption probability at the origin, $q$, and the drift velocity towards the origin, $v$, were assumed constant. Thus initially we can assume this lattice expression in our model as well,

$$
a(0)=\frac{q}{(1-q) \ell}
$$

where we neglected the higher-order terms in $\ell$.

Quite generally for the coagulation reaction $A+A \rightarrow A$, one anticipates that the density (1.1) approaches a universal power-law behavior, independent of the initial density and of the reaction probability $q$,

$$
c(\tau \text {-large })=\frac{1}{\sqrt{\pi \tau}}
$$

where the universal coefficient has been calculated [8] for the case of instantaneous reaction, $q=1$, corresponding to $a=\infty$ in the radiation boundary conditions $(3.1)$. Note that in this limiting full adsorption case $P(0, t>0)=0$ and the problem is just the ordinary diffusion on half-line $x \geq 0$ because there is no hard-core induced "drift" current.

Our numerical Monte Carlo studies, details of which will be reported in Section 5, support the expectation of the universal coefficient in (3.4). A simplistic line of argument for this universality appeals to the recurrence property of the one-dimensional 
random walk. Once two particles meet at late times when the system is dilute, they will have many additional encounters with each other before being "mixed" by diffusion with other surrounding particles which are distance of order $1 / c(t)$ away. The partial reaction probability is thus expected to be effectively "renormalized" to 1.

Note however that the same two-particle correlation that leads to these repeated encounters also perturbs the density locally. In fact, in the problem of two particles (one interparticle distance) which coagulate with partial reaction probability, the average particle number, initially 2, exceeds the final, infinite-time value 1 by an amount proportional to $1 / \sqrt{\tau}$ for large times, with a nonuniversal coefficient. Thus the universality of the coefficient in (3.4) is unlikely to be explained at the two-particle level only; it must be attributed to many-body fluctuation effects. An important conclusion is that the quantity $a(t)$ in the radiation boundary conditions must be forced to diverge phenomenologically. It cannot get "renormalized" dynamically within the two-particle relative-distance diffusive model; the $t$-dependence of $a(t)$ must be introduced by hand.

In our calculations we took

$$
a(t)=a(0) \rho / c(t)
$$

This choice was favored because it yielded a convenient closed calculation scheme, to be specified shortly. It is also dimensionally correct. Furthermore, the effective reaction probability $q_{\text {effective }}(t)$, implied by the choice (3.5), approaches 1 as $\sim 1 / \sqrt{t}$, which seems reasonable in view of the simplistic two-particle argument presented above. 


\section{$\underline{\text { 4. Solution within the Adiabatic Approximation }}$}

The set of relations (2.1), (2.2), (2.3), (2.10), (2.11), (3.1), (3.5), should be solved for $P(x, t)$ and $c(t)$. In order to make the calculation tractable, and given the fact that this whole treatment is not exact but just an approximation scheme, we will treat the problem adiabatically. Thus, we first solve (2.10) with constant $p$, supplemented by the boundary conditions (3.1) with constant $a$. Given the initial condition (2.3), the resulting problem is solved for $P(x, t ; p, a)$. The relations $(2.2),(2.11),(3.5)$, evaluated with this "adiabatic" $P$-function, provide a set of three equations for the quantities $p$, $a, c$, as functions of time.

It is convenient to define the quantity

$$
b=(1-q) p .
$$

It is also useful to recall the relation

$$
a=\frac{q \rho}{(1-q) \ell c},
$$

which follows from (3.3) and (3.5). Thus we solve the diffusion equation

$$
\frac{\partial P}{\partial \tau}=b \frac{\partial P}{\partial x}+\frac{\partial^{2} P}{\partial x^{2}},
$$

with the initial condition (2.3) and the boundary conditions at $x=0$ given by

$$
\begin{gathered}
(a-b) P(0, \tau)=\frac{\partial P}{\partial x}(0, \tau) . \\
-12-
\end{gathered}
$$


The latter relation follows from (2.8) and (3.1).

The solution $P(x, \tau ; a, b)$ is obtained with fixed $a$ and $b$. Rather cumbersome calculations, not presented in detail here, yield the Laplace Transform of this function in the time variable $\tau$. It turns out that the inverse Laplace Transform of $P(0, \tau ; a, b)$ can be obtained in closed form. The result can then be used to write, by (2.11), the equation

$\frac{\rho-a}{\rho} p=\left(\rho-\frac{b}{2}\right) e^{\rho(\rho-b) \tau} \operatorname{erfc}\left[\left(\rho-\frac{b}{2}\right) \sqrt{\tau}\right]-\left(a-\frac{b}{2}\right) e^{a(a-b) \tau} \operatorname{erfc}\left[\left(a-\frac{b}{2}\right) \sqrt{\tau}\right]$.

Note that we essentially have two unknowns, $p$ and $c$, since $b$ and $a$ are simply related to $p$ and $c$, respectively. The second equation connecting these two unknowns follows from (2.2). In conventional diffusion problems, relation (2.4) for the probability current can be utilized to reduce the time-derivative of (2.2) to the form involving flux at the origin,

$$
\frac{d c}{d t}=\rho J(0, t)
$$

where $J(0, t)$ can be further replaced by $-2 D a(t) P(0, t)$, via (3.1). The derivation of (4.6) would remain valid for our more complicated system of relations had we treated it exactly. However, it is not ensured a priori that the approximate functions, obtained within our adiabatic scheme, identically satisfy (4.6). Thus despite its suggestive simplicity, the relation

$$
\frac{d c(t)}{d \tau}=-\rho a p
$$

which follows from (4.6), was not utilized within our adiabatic solution scheme. We preferred to use directly the integral form (2.2) which is presumably more "robust" in 
preserving physically reasonable properties when setting up approximations (see further comments in Section 5).

Fortunately, the Laplace Transform of the integral on the left-hand side of (2.2), calculated within the adiabatic approximation, can be inverted directly. Again due to complexity of the intermediate expressions we only quote the final result, which was further simplified by using (4.5):

$$
\frac{b-a}{\rho} c=p+\frac{a+\rho-b}{b-\rho}\left\{\left(\rho-\frac{b}{2}\right) e^{\rho(\rho-b) \tau} \operatorname{erfc}\left[\left(\rho-\frac{b}{2}\right) \sqrt{\tau}\right]-\frac{b}{2} \operatorname{erfc}\left(\frac{b}{2} \sqrt{\tau}\right)\right\} .
$$

Relations (4.5) and (4.8) determine the two unknowns $p$ and $c$ as functions of $\tau$. They involve a standard error function,

$$
\operatorname{erfc}(y)=\frac{2}{\sqrt{\pi}} \int_{y}^{\infty} e^{-z^{2}} d z
$$

and can be solved straightforwardly to numerically obtain the plot of, for instance, $c(\tau)$, and well as closed-form analytical expressions in various limits. 


\section{$\underline{\text { 5. Results and Discussion }}$}

Numerical Monte Carlo data for the coagulation reaction with partial reaction probability were obtained for periodic lattices of size $10^{5}$. The lattice was randomly populated with the initial density of particles, $\rho \ell$ per site. Then the particles hopped randomly and independently, with the rate of the hopping attempts selected to keep the fixed single-particle (dilute-limit) diffusion constant, $D$, value. When a particle attempted to hop to an empty target (nearest neighbor) site, it was simply moved there. However if the target site happened to be occupied, the attempting particle was left in the original site with probability $1-q$, or it was removed (representing coagulation at the target site) with probability $q$. The results for the density were recorded and averaged over $10^{3}$ independent Monte Carlo runs.

Figure 2 illustrates two typical situations. In the case of relatively large initial density and slow reaction, i.e., when the system takes a long time to reach continuumlimiting behavior, the approximation is semiquantitative at intermediate times although it is quite accurate for short times, and it reproduces the correct universal asymptotic behavior at large times. The latter is not seen in Figure 2, but it can be evaluated analytically; see below.

In the case of low initial density and fast reaction, the continuum limiting behavior sets in at earlier times. Our approximation then follows the data quantitatively, although there is a small deviation at intermediate times. In fact such small deviations are present also in the case of the instantaneous reaction [8] which is the optimal continuum-limiting situation (see further comments below) for modeling by the diffusion equation.

For short times, relations (4.5) and (4.8) suggest that the functions $c(\tau)$ and $p(\tau)$ 
can be expended in the Taylor series in $\sqrt{\tau}$. For instance,

$$
p=\rho-\frac{2 q \rho}{(1-q) \ell}[1+(1-q) \ell \rho] \sqrt{\frac{\tau}{\pi}}+O(\tau) .
$$

The derivation of all but the leading terms in this and other expansions reported in this section was carried out in Mathematica, a symbolic computer language. Specifically, the $O(\tau)$ term in (5.1) has a nonzero, but rather complicated coefficient.

For the density at short times, we derived the expansion

$$
c=\rho-\frac{q \rho^{2}}{(1-q) \ell} \tau+\frac{4 q^{2} \rho^{2}}{3 \sqrt{\pi}(1-q)^{2} \ell^{2}}[1+(1-q) \ell \rho] \tau^{3 / 2}+O\left(\tau^{2}\right) .
$$

The absence of the $O\left(\tau^{1 / 2}\right)$ term in (5.2) is interesting. Indeed, let us consider the mean-field rate equation,

$$
\frac{d c}{d \tau}=-\Gamma c^{2}
$$

where the rate constant is denoted by $\Gamma$. The solution is

$$
c=\frac{\rho}{1+\Gamma \rho \tau}=\rho-\Gamma \rho^{2} \tau+\Gamma^{2} \rho^{3} \tau^{2}+O\left(\tau^{3}\right)
$$

Thus the expansion (5.2) suggests that the mean-field behavior applies "initially," in that the density has the mean-field slope, corresponding to the rate constant

$$
\Gamma=\frac{q}{(1-q) \ell}=a(0)
$$

where the latter expression probably applies more generally than for our particular choice of $a(t)$; cf. relations $(3.3),(3.5),(4.7)$. In fact, the form of (4.7) suggests that 
the mean-field approximation which amounts to assuming that the probability of two particles to react is proportional to $c^{2}$, in fact takes the form

$$
P(0, t) \simeq P_{\text {mean-field }}(0, t)=\frac{c^{2}(t)}{\rho}
$$

Note that the short-time expansion coefficients diverge as $q \rightarrow 1$. In fact our expression for $c(\tau)$ then coincides with the simple-diffusion results [8] for the instantaneousreaction case. The mean-field approximation then breaks down for all $t \geq 0$; see [8] for details.

We now turn to the large-time behavior. The functions $p(\tau)$ and $c(\tau)$ can be expanded in asymptotic series in powers of $\tau^{-1 / 2}$ in this limit. Not surprisingly, the expansion is ill-defined for $q=0$. Assuming $q>0$, we derived the first two leading terms,

$$
\begin{gathered}
c(\tau) \simeq \frac{1}{\sqrt{\pi \tau}}+\frac{(1-q) \ell}{\pi q \tau}, \\
p(\tau) \simeq \frac{(1-q) \ell}{2 \pi \rho^{2} q \tau^{2}}+\frac{(1-q)^{2} \ell^{2}}{\rho^{2} q^{2} \pi^{3 / 2} \tau^{5 / 2}} .
\end{gathered}
$$

The leading term in $c(\tau)$ is universal as expected.

Finally, both for short times and for large times, we checked that to order $\tau$ and $1 / \tau^{2}$, in the respective expansions, the relation (4.7) in not violated by the adiabaticapproximation results, i.e., the difference of the left- and right-hand sides is of order higher than the powers indicated. However, no careful numerical or analytical tests were attempted at intermediate times.

In summary we proposed an analytical approximation scheme to describe the crossover from the initially mean-field to asymptotically fluctuation-dominated behav- 
ior in one-dimensional two-particle coagulation reactions. The resulting expressions are not simple. However, they suggest the form of the small- and large-time behaviors, i.e., the appropriate variable combinations which are presumably quite general. They also indicate that any "scaling-form" description of the crossover must allow for at least two scaling combinations,

$$
\rho \sqrt{D t} \quad \text { and } \quad \frac{q \sqrt{D t}}{(1-q) \ell} \text {. }
$$

The approximation scheme used in this work is not an obvious "leading order" in a systematic expansion but rather a phenomenological closed-form model. It is hoped that this first theoretical study will stimulate further work on partial-reaction-probability diffusion-reaction systems and generally on the mean-field to fluctuation-dominated crossover in nonequilibrium dynamical models.

This research has been supported in part by the NSF grants PHY-8958506, DMR9023541 and PHY-9214715. 


\section{REFERENCES}

[1] M. Bramson and D. Griffeath, Ann. Prob. 8, 183 (1980).

[2] D.C. Torney and H.M. McConnell, J. Phys. Chem. 87, 1941 (1983).

[3] K. Kang, P. Meakin, J.H. Oh and S. Redner, J. Phys. A17, L665 (1984).

[4] T. Liggett, Interacting Particle Systems (Springer-Verlag, New York, 1985).

[5] Z. Racz, Phys. Rev. Lett. 55, 1707 (1985).

[6] A.A. Lushnikov, Phys. Lett. A120, 135 (1987).

[7] M. Bramson and J.L. Lebowitz, Phys. Rev. Lett. 61, 2397 (1988).

[8] C.R. Doering and D. ben-Avraham, Phys. Rev. A38, 3035 (1988).

[9] V. Kuzovkov and E. Kotomin, Rep. Prog. Phys. 51, 1479 (1988).

[10] J.L. Spouge, Phys. Rev. Lett. 60, 871 (1988).

[11] J.G. Amar and F. Family, Phys. Rev. A41, 3258 (1990).

[12] D. ben-Avraham, M.A. Burschka and C.R. Doering, J. Stat. Phys. 60, 695 (1990).

[13] V. Privman, J. Stat. Phys. 69, 629 (1992).

[14] R. Kroon, H. Fleurent and R. Sprik, Diffusion-Limited Exciton Fusion Reaction in One-Dimensional Tetramethylammonium Manganese Trichloride (TMMC), Phys. Rev. E (in print).

[15] J.C. Lin, C.R. Doering and D. ben-Avraham, Chem. Phys. 146, 355 (1990).

[16] L. Braunstein, H.O. Martin, M.D. Grynberg and H.E. Roman, J. Phys. A25, L255 (1992).

[17] H.O. Martin and L. Braunstein, Study of $A+A \rightarrow 0$ with Probability of Reaction 
and Diffusion in One Dimension and in Fractal Substrata, preprint.

[18] V. Privman and P. Nielaba, Europhys. Lett. 18, 673 (1992).

[19] P. Seglar and M.E. Fisher, J. Phys. C13, 6613 (1980).

[20] V. Privman and M.D. Grynberg, J. Phys. A25, 6575 (1992).

[21] H. Teitelbaum, S. Havlin and G. Weiss, Chem. Phys. 146, 351 (1990).

[22] H. Teitelbaum, R. Kopelman, G. Weiss and S. Havlin, Phys. Rev. A41, 3116 (1990).

[23] F.C. Collins and G.E. Kimball, J. Colloid Sci. 4, 425 (1949).

[24] J.C. Lin, Phys. Rev. A45, 3892 (1992). 


\section{FIGURE CAPTIONS}

Fig. 1. A schematic representation of the diffusive motion of four particles. When two middle particles run into each other but do not react, their interparticle spacing, $x_{m}$, bounces off zero, while the nearby interparticle distances, $x_{m \pm 1}$, receive a "push" towards zero.

Fig. 2. Numerical Monte Carlo results, solid lines, compared to the approximate density $c(\tau)$ calculated from (4.5) and (4.8), dashed lines. The upper curves correspond to a typical case of initially strong lattice effects, $\rho=0.8$ and $q=0.1$ (large initial density and slow reaction), while the lower curves correspond to the case of nearcontinuum initial conditions, $\rho=0.1$ and $q=0.9$, i.e., low density and fast reaction. The latter data were multiplied by 2 for better resolution. The densities are in units of $1 / \ell$, while $\tau$ is measured in units of $\ell^{2}$. 